\title{
The Design of the Monitoring and Evaluation System of the Reservoir Resettlement Later-period Supportive Policy's Implementation Effect
}

\author{
Xin $\mathrm{XU}^{\mathrm{a}}$, Xiao-Yi WANG ${ }^{\mathrm{b},{ }^{*},}$, Yu-Ting BAl ${ }^{\mathrm{c}}$, Ying-Qiang WANG ${ }^{\mathrm{d}}$, Ji-Ping $\mathrm{XU}^{\mathrm{e}}$ \\ Beijing Technology and Business University, Beijing 100048, China \\ abjgsdxxx@126.com, bsdwangxy@163.com, ${ }^{\text {bbyting@foxmail.com, }{ }^{d} x i a o q i a n g a a a 2007 @ 163 . c o m, ~}$ \\ exujiping@139.com \\ ${ }^{*}$ Corresponding author
}

Keywords: Reservoir resettlement, Later-period supportive policy, Monitoring and evaluation, System design.

\begin{abstract}
According to the characteristics of the monitoring and evaluation of the reservoir resettlement later-period supportive policy's implementation effect, the comprehensive evaluation method based on AHP was proposed. The framework and modules of the monitoring and evaluation system were designed and the platform was built up. The system which provides decision-making platform for government department has achieved the functions such as the questionnaires' entering and management, statistical analysis, comprehensive evaluation and system settings.
\end{abstract}

\section{Introduction}

Reservoir resettlement, a kind of immigration phenomenon caused by the comprehensive utilization, exploitation and governance of the water resource, is a large and complex system engineering referring to the politics, economy, society, population, resources, environment and other fields[1]. The later-period supportive policy of reservoir resettlement is aimed at helping the immigrants who have been arranged to recovery their production and living after being made proper compensations to their property damage. The comprehensive and scientific evaluation on the implementation effect of the later-period supportive policy of reservoir resettlement helps the government to realize the implementation of the policy on the whole and provides the decision basis of the policy adjustment[2].

The study on the comprehensive evaluation and system design of the reservoir resettlement later-period supportive policy has been achieved. Wang Qingfa made the comprehensive evaluation on the Jiangsu province's reservoir resettlement using the method of AHP-FCE[3]. Du Ruifang did the risk assessment on the Henan province's implementation effect of the reservoir resettlement based on the BP neural network model[4]. Gao Wei built the information management system of Sichuan province's reservoir resettlement[5]. Chen Chunqin developed the evaluation system of the reservoir in Jiangxi province adopting the layering development mode based on Ruby on Rails[6]. However the indicator system and the evaluation method in the existing researches are different. And the related systems mainly realize information management, lacking the support for the comprehensive evaluation function. In order to solve the above problem, the paper established the implementation effect evaluation method and monitoring and evaluation system of the reservoir resettlement later-period supportive policy, realizing the monitoring and evaluation of information management and the comprehensive evaluation function.

\section{The Comprehensive Evaluation Method Based on AHP}

\section{The Comprehensive Evaluation Method}

Analytic hierarchy process (AHP) is a kind of classic system engineering analysis method from qualitative to quantitative [7]. According to the issue of the monitoring and evaluation of the reservoir resettlement in Beijing [8], the comprehensive evaluation method based on AHP is proposed and the concrete steps are given as follows: 
(1) Build the evaluation index of the later-period supportive policy's implementation effect;

(2) Build the hierarchical structure of the policy implementation effect evaluation;

(3) Quantize the corresponding judgment matrix made up of index, and the characteristic vector method is used to determine evaluation index weights. Then judge the consistency;

(4) Build each index's judgment matrix corresponding to the evaluation, and calculate the corresponding weight;

(5) Calculate the comprehensive weights of evaluation indexes, setting them as a basis for the final optimization index.

\section{The Implementation of Evaluation}

According to the project requirements of the monitoring and evaluation on the reservoir resettlement later-period supportive policy's implementation effect, the AHP evaluation method is used to determine the weights of the index in which the reservoir immigrants of Beijing's districts have been set as the objects of study. Firstly the relative importance judgment matrix between each index has been determined through expert advice law. The calculation results are shown in Table 1 in which the secondary indexes (such as the roads, electricity, water condition, medical conditions, culture and education) on the production and life are set as the algorithm example.

Table 1 The relative importance judgment matrix between secondary indexes

\begin{tabular}{|c|c|c|c|c|c|c|}
\hline $\mathrm{C}$ & $\mathrm{C} 1$ & $\mathrm{C} 2$ & $\mathrm{C} 3$ & $\mathrm{C} 4$ & $\mathrm{C} 5$ & Weight $\mathrm{w}$ \\
\hline $\mathrm{C} 1$ & 1 & 3 & $1 / 3$ & 5 & 6 & 0.2663 \\
\hline $\mathrm{C} 2$ & $1 / 3$ & 1 & $1 / 5$ & 3 & 6 & 0.1399 \\
\hline $\mathrm{C} 3$ & 3 & 5 & 1 & 6 & 7 & 0.4896 \\
\hline $\mathrm{C} 4$ & $1 / 5$ & $1 / 3$ & $1 / 6$ & 1 & 3 & 0.0683 \\
\hline $\mathrm{C} 5$ & $1 / 6$ & $1 / 6$ & $1 / 7$ & $1 / 3$ & 1 & 0.0358 \\
\hline
\end{tabular}

The comprehensive evaluation on the immigrations' production and life is made based on the weights of indexes. The evaluation results were 0.0267 (2006, before the later-period supportive policy was carried out) and 0.03738 (2010, after the policy was carried out) respectively. The analysis combined with the actual situation is as follows: the samples villages' roads are in good condition, and the road hardening rate has increased. Sample villages have realized the electricity and the safe drinking water, and the immigration is satisfactory on the water quality and supply. The new rural cooperative medical coverage rate is high, and the immigration is satisfied with the conditions for medical treatment on the whole. Immigrants' education is in good condition, and the villages have the relevant cultural facilities, which provide a good condition for the rich culture life.

\section{The Integrated Design of the Monitoring and Evaluation System}

The monitoring and evaluation system of the reservoir resettlement later-period supportive policy's implementation effect encapsulates the different functions into multiple subsystems. The structure can improve the system's running speed and reduce the maintenance workload. The system platform modular structure is shown in Fig. 1. 


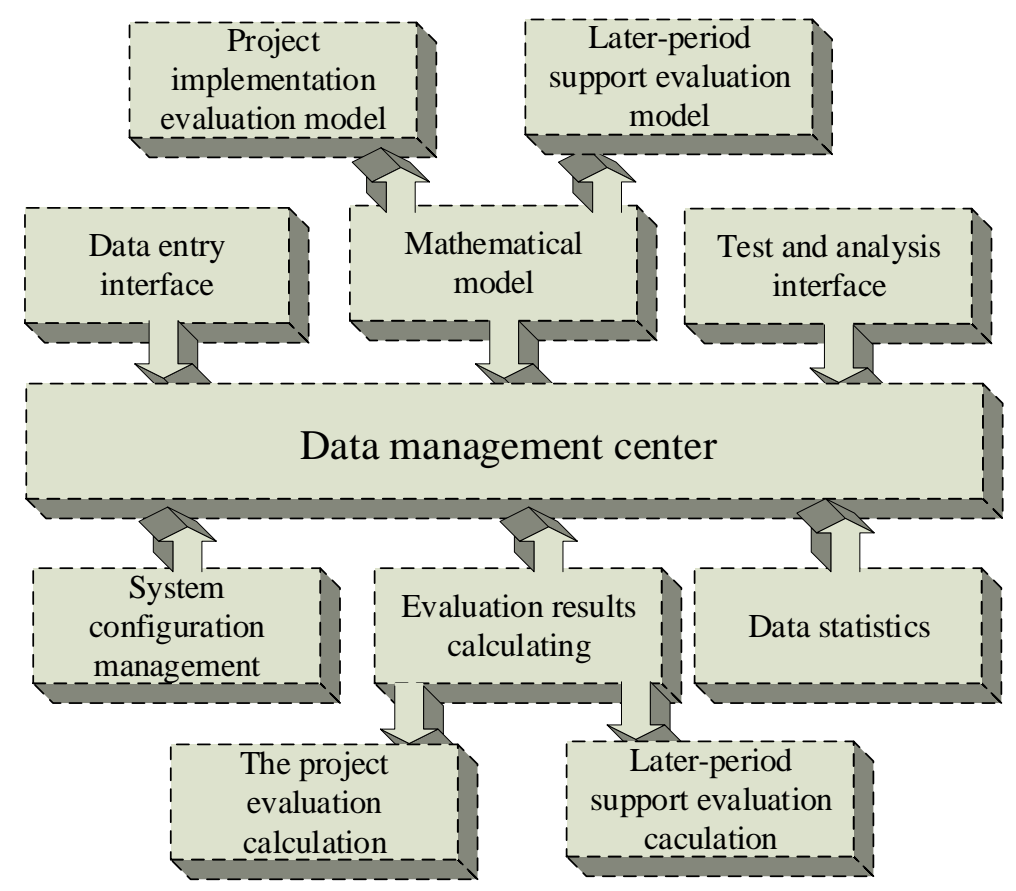

Fig. 1 The system platform modular structure

The system platform's specific function of each subsystem is as follows:

(1) Data management center. The user input window function is realized through data entry interface, which can input data simply and quickly, at the same time it provides data query and modify functions. Using database technology, it can storage all the raw data, the calculation results, the final evaluation results, system management data and so on. It is the linkage of other modules.

(2) Mathematical model. It is integrated with the analysis model of later-period supportive policy and project implementation, and it has the functions such as data transformation, model encapsulation, etc. The encapsulated model provides a unified interface, convenient for other subsystems calling analysis calculation and statistics.

(3) Test and analysis interface. The interface is mainly used for internal testing, specially providing to the testers before the system is released, convenient for testing the system operation ability, computing precision and stability performance. The purpose is to find out the loopholes in the system and to realize the system perfectly.

(4) System configuration management. It's the auxiliary type module of the system operation, mainly including user management, permissions setting, interface style settings and related functions.

(5) Evaluation results calculating. It mainly coalesces the data management center module and the mathematical model module, so as to assess the implementation effect of later-period supportive policy accurately.

(6) Data statistics. It provides users with the different forms of query statistics feature, by using the graphical representation, to display data contrast and analysis results intuitively.

\section{The Implementation of Monitoring and Evaluation System}

In view of the reservoir resettlement later-period supportive policy's monitoring and evaluation requires large amount of information, the system platform need to meet the requirements such as huge amount of data, fast computing speed and high precision. The system is built by using high-performance server hardware platform, based on Visual Studio 2010 programming environment, using $\mathrm{C}++$ programming language, integrated SQL2005 database technology. The system implements the functions of questionnaire data entry, data statistics and analysis, effect evaluation, user management, etc. The main interface includes the menu bar, toolbar, classification show bar and the primary displaying area, shown in Fig. 2. In order to introduce the system 
accurately, the interfaces in this paper have all been translated to English from Chinese.

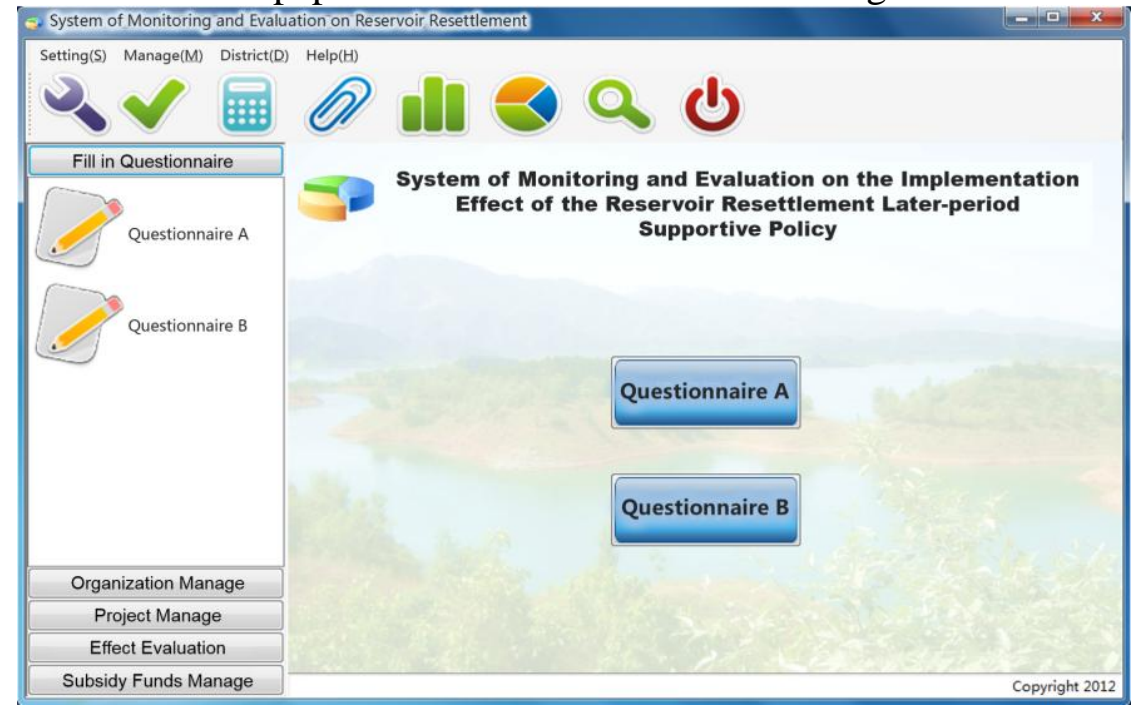

Fig. 2 The main interface

The menu bar includes four parts: Setting, Manage, District and Help. Toolbar provides shortcuts for the functional operation, including the database Settings, data add, search, data analysis, comprehensive evaluation and other functions. Classification show bar shows the evaluation index system, such as organization management, project management, subsidy funds management, etc. The primary area can display interfaces according to the different operating.

(1) The questionnaire data entry and management

The questionnaire of this system is divided into questionnaire A and questionnaire $\mathrm{B}$. Among them, questionnaire $\mathrm{A}$ is aimed at immigration individual, questionnaire $\mathrm{B}$ is aimed at monitoring the village's basic situation. Questionnaire input interface is shown in Fig. 3 (questionnaire A, for example). Filling in the questionnaire need to complete the radio, multiple-choice, blanks and so on. Questionnaire data management (Fig. 4) can realize the view and management functions of the questionnaires filled out for different years and areas.

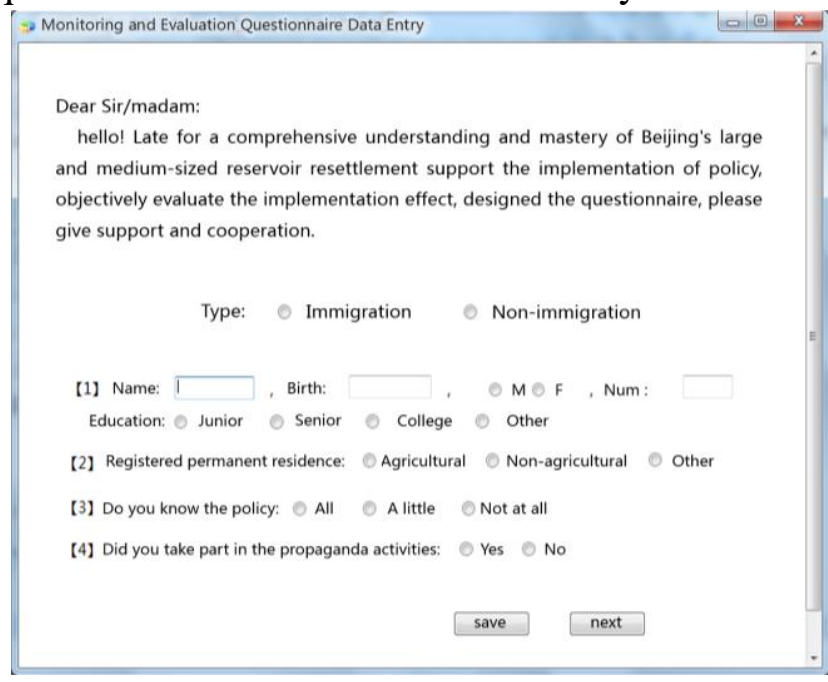

Fig. 3 The questionnaire input interface

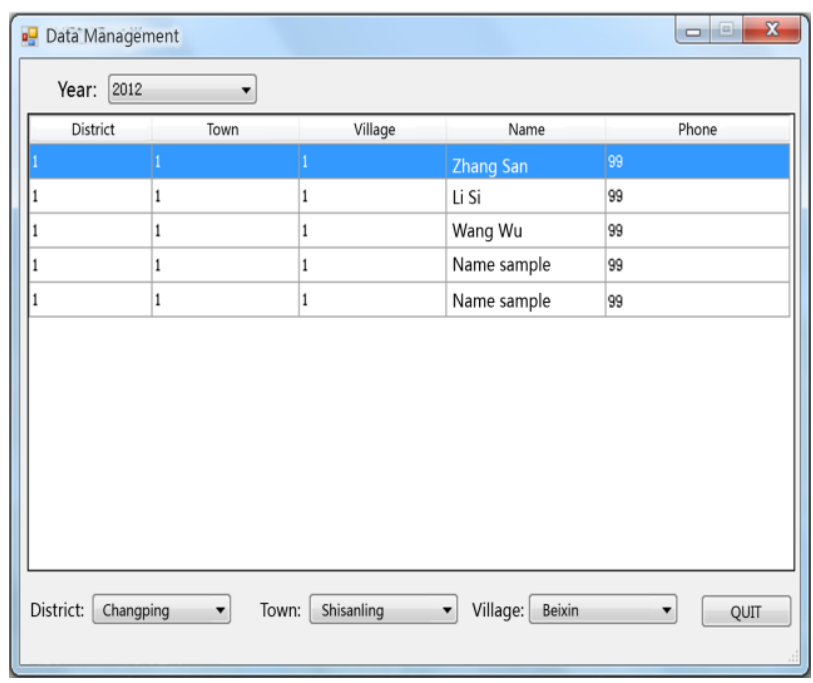

Fig. 4 The data management interface

(2) The data statistics

The function can display the data vividly through choosing the type of questionnaire and statistics, according to the demand of different levels and styles (Fig. 5).

(3) The comprehensive evaluation

The system is integrated with the comprehensive evaluation method of this paper, which can 
realize the synthetical evaluation of different areas, vintage and evaluation index. Based on the AHP method, users can modify the index weights which has been programmed in the software (Fig. 6), ensuring the rationality and validity of the algorithm.

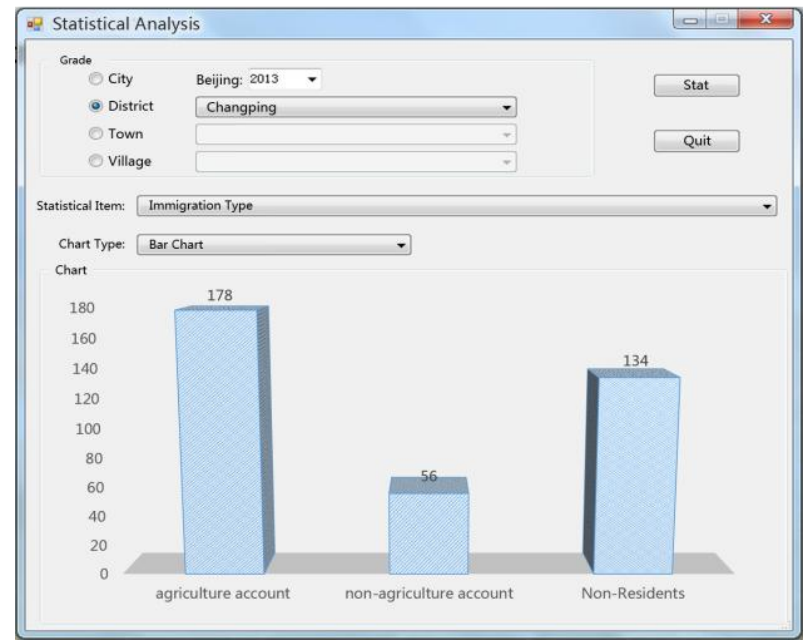

Fig. 5 The statistical analysis interface

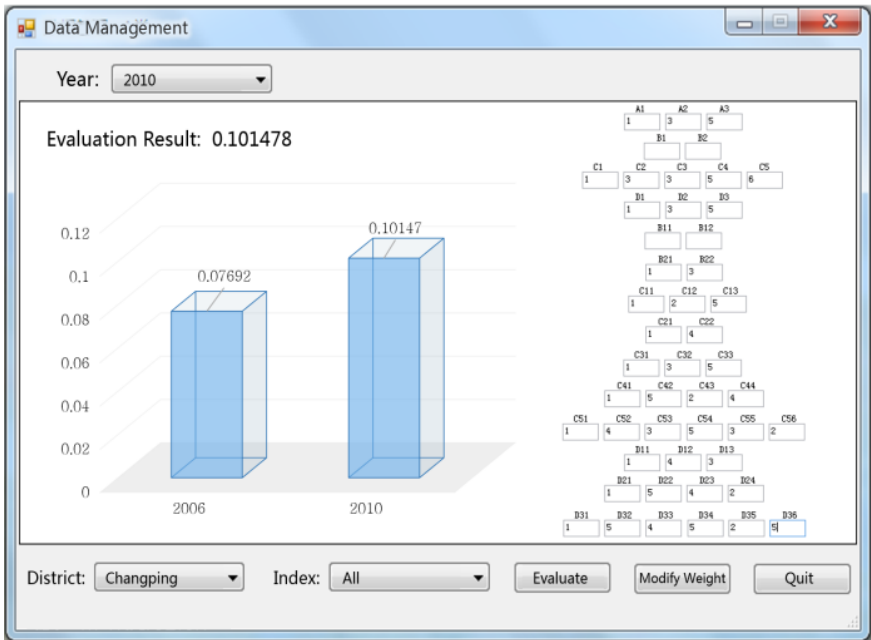

Fig. 6 The comprehensive evaluation interface

(4) The system auxiliary function

District information maintenance: It can realize the encoding management of the subordinate districts towns and villages, guarantying the accuracy and integrity of the data stored (Fig. 7).

User management: Super administrators can add, delete, modify the user information, and match these permissions for users at different levels, to ensure the security of data and the traceability of system operation (Fig. 8).

System settings: It can deploy the configuration information in database and server system. The system also provides "theme switch" function, to provide users with good man-machine interaction experience.

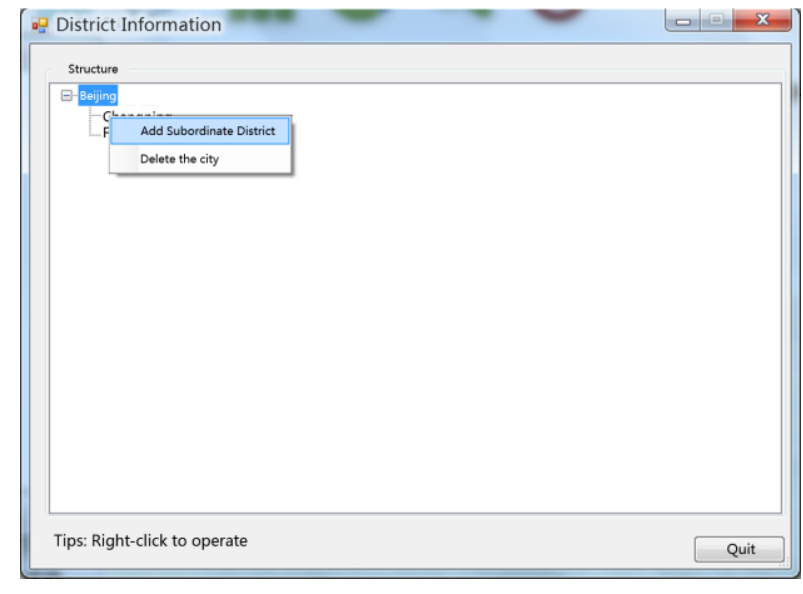

Fig. 7 The district information interface

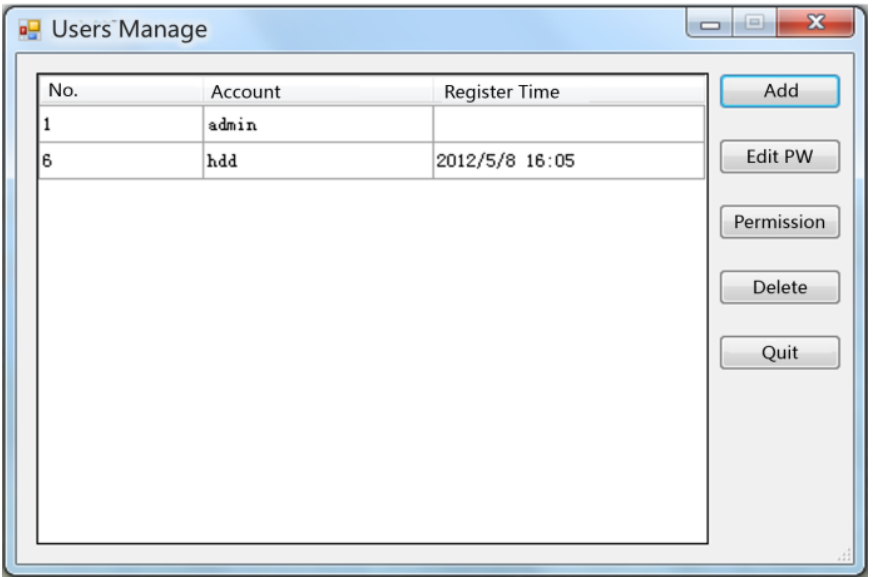

Fig. 8 The user management interface

\section{Summary}

The paper proposed the comprehensive evaluation method of the reservoir resettlement later-period supportive policy's implementation effect based on AHP practically. And the monitoring and evaluation system was built up which can improve the information management efficiency of the reservoir resettlement later-period supportive policy's implementation. It will help enhancing the data security and improving the capacity of monitoring and evaluation, providing the decision basis for the government sector at the same time. 


\section{Acknowledgement}

This study is supported by The Innovation Ability Promotion Project of Beijing Municipal Commission of Education College (PXM2013-014213-000098, PXM2014-014213-000033), High-level Talent Introduction and Training Program of Beijing Municipal Colleges and Universities (CIT\&TCD201404031). Those supports are gratefully acknowledged.

\section{References}

[1] Zhang Baoxin. The Theory and Practice of Developmental Resettlement [M]. Beijing: China Three Gorges Publishing House, 1999.

[2] Yang Jianshe, Zuo Ping. The Monitoring and Evaluation on the Implementation of the Reservoir Resettlement Later-period Supportive Policy [J]. Water Resources Planning and Design, 2009: 44-45.

[3] Wang Qingfa, Sun Zhonggen. The Comprehensive Evaluation on the Reservoir Resettlement Later-period Supportive Implementation Based on AHP-FCE [J]. Jiangsu Water Conservancy, 2012(7): 11-13.

[4] Du Ruifang, Yao Kaiwen. The Risk Assessment of the Reservoir Resettlement Support Based on BP Neural Network Model [J]. Water Resources and Power, 2014, 32(1): 153-156.

[5] Gao Wei, Fan Guangwei, Shen Zheng, et al. The Design and Implementation of Management Information System of Sichuan Province Reservoir Resettlement Later-period Support [J]. China Water Transport, 2013(9): 80-81.

[6] Chen Chunqin. The Development of Evaluation System of Reservoir Later-period Support Based on RoR [D]. Jiangxi: Jiangxi Agricultural University, 2013.

[7] Saaty T L. Analytic hierarchy process [M]. Encyclopedia of Operations Research and Management Science. Springer US, 2013: 52-64.

[8] Xu Xin, Wang Xiaoyi, Xu Jiping, et al. Evaluation on Implementation Effect of Later-Period Supportive Policy of AHP-based Reservoir Resettlement [C]. Contemporary Innovation and Development in Statistical Science. Australia: Aussino Academic Publishing House, 2012: 808-812. 